\title{
A Distributed Particle Filter for Acoustic Source Tracking Using an Acoustic Vector Sensor Network
}

\author{
Xionghu Zhong ${ }^{\# 1}$, Arash Mohammadi ${ }^{\dagger 2}$, A. B. Premkumar ${ }^{\star 3}$, Amir Asif ${ }^{\dagger 4}$ \\ \# School of Computer Engineering, College of Engineering, Nanyang Technological University, Singapore, 639798. \\ 1 xhzhong@ntu.edu.sg \\ * Engineering Department, Faculty of Engineering, University of Malaya, KualaLumpur, Malaysia \\ 3 benjamin.premkumar@gmail.com \\ $\dagger$ Department of Computer Science and Engineering, York University, Toronto, Canada, M3J 1P3. \\ 2 marashecse.yorku.ca, ${ }^{4}$ asif@cse.yorku.ca
}

\begin{abstract}
This letter presents a distributed particle filtering (PF) approach for wideband acoustic source tracking using an acoustic vector sensor (AVS) network. At each distributed AVS node of the AVS network, the unscented information PF (UIPF) provides local estimates of the source location. A distributed consensus algorithm, based on the first and second order statistics of the localized UIPF estimated states, then obtains the global position estimate for the overall AVS network. Since the UIPF inverts smaller order matrices, its computational complexity is significantly less as compared to its centralized counterpart. The performance of the proposed tracking algorithm is studied under both single source and multiple source tracking scenarios.
\end{abstract}

Index Terms-Acoustic vector sensor, sensor network, particle filtering, multiple source tracking, unscented information filter.

\section{INTRODUCTION}

Acoustic vector sensor (AVS) employs a co-located sensor structure capable of providing 2-D (azimuth and elevation) direction of arrival (DOA) information. The AVS manifold is independent of source signal's frequency, which enhances its utility in wideband acoustic signal processing applications [1], including acoustic source detection [2], localization [1] and tracking [3], [4] in underwater, battlefield, and room acoustic environments [5], [6]. More recent AVS applications include speech enhancement for hands free communication, sound source localization in air, and detection of seismic activities.

This letter presents a distributed wideband acoustic source tracking algorithm for the distributed AVS network. A diffusive estimation scheme, namely the distributed unscented information particle filter (dUIPF), is developed. The proposed approach consists of three main steps: (i) At each AVS node, an unscented information filter (UIF) [7] is introduced to approximate the optimum importance function to draw the particles according to both current measurements and previous state estimates; (ii) Local particle filters are applied at each node to estimate the first order and the second order statistics for the localized state estimates, and; (iii) Each node diffuses its local statistics throughout the network by communicating with nodes within its local neighborhood. A consensus filter is employed in step (iii) to fuse these local statistics to obtain the global estimates. With the proposed dUIPF, the positions of multiple wideband acoustic sources can be estimated directly from the received AVS signals. There is no need to preprocess the received signals to extract the DOA measurements, nor to associate the DOA measurements to each source. Further, the information communicated within the AVS network is limited to the first and second order statistics of the local estimates keeping the communication overhead manageable.

In the past, different centralized approaches were developed for AVS based localization and tracking applications. In [1], for example, the Capon beamforming algorithm is used at each node to estimate the local DOAs associated with the target. The local nodes communicate the processed DOAs to the central processor $(\mathrm{CP})$, where the position of the source is triangulated using the least squares (LS) approach. Such DOA based approaches, including [8] developed for distributed microphone array based acoustic source tracking, are, however, not applicable to multiple sources and 3-D source tracking applications. Further, their performance is dependent on the accuracy of the DOA estimates. Among the multiple source tracking approaches, the centralized extended information based PF (cEIPF) [9] is promising. But its applicability to large distributed networks without a $\mathrm{CP}$ is somewhat limited. Our dUIPF approach belongs to the distributed category of AVS trackers and has several differences from [1] and [9]. Compared to the reweighted LS (RLS) approach [1], the dUIPF achieves superior performance in both singlesource and multiple-source tracking scenarios. Compared to the cEIPF [9], both computation and communication cost are substantially reduced. The rest of this letter is organized as follows. In Section II, the AVS signal model is introduced. Section III presents the localized implementation of the dUIPF at each AVS node. Consensus based information fusion used to integrate local state statistics to derive the global estimate is introduced in Section IV. Different simulations are organized in Section V with the letter concluded in Section VI.

\section{AVS SIGNAL MODEL}

To track $M$ acoustic sources located at $\mathbf{x}_{m, k}=$ $\left[x_{m, k}, y_{m, k}, z_{m, k}\right]^{T} \in \mathbb{R}^{3 \times 1}$, for $(1 \leq m \leq M)$, at time instant $k$, assume $N$ AVSs at fixed locations $\mathbf{x}_{0}^{n}=\left[x_{0}^{n}, y_{0}^{n}, z_{0}^{n}\right]^{T} \in$ $\mathbb{R}^{3 \times 1}$, for $(1 \leq n \leq N)$, are arbitrarily deployed. The DOA of 
the acoustic signal associated with the $m$ th source at the $n$th AVS is

$$
\begin{aligned}
& \phi_{m, k}^{n}=\tan ^{-1}\left(\frac{x_{m, k}-x_{0}^{n}}{y_{m, k}-y_{0}^{n}}\right), \\
& \psi_{m, k}^{n}=\tan ^{-1}\left(\frac{z_{m, k}-z_{0}^{n}}{\sqrt{\left(x_{m, k}-x_{0}^{n}\right)^{2}+\left(y_{m, k}-y_{0}^{n}\right)^{2}}}\right),
\end{aligned}
$$

where $\phi_{m, k}^{n} \in(-\pi, \pi]$ and $\psi_{m, k}^{n} \in[-\pi / 2, \pi / 2]$ represent the azimuth angle and the elevation angle respectively, and superscript $T$ denotes the transpose operator.

Let $\boldsymbol{u}_{m, k}^{n}=\left[\cos \psi_{m, k}^{n} \cos \phi_{m, k}^{n}, \cos \psi_{m, k}^{n} \sin \phi_{m, k}^{n}, \sin \psi_{m, k}^{n}\right]^{T}$ be the unit direction vector pointing out from the $n$th sensor towards the $m$ th source. Assuming that at time step $k$, $T_{0}$ number of snapshots are considered, the collection of wideband source signals $\mathbf{s}_{m}(k),(1 \leq m \leq M)$, is given by $\mathbf{S}_{k}=\left[\mathbf{s}_{1}(k), \ldots, \mathbf{s}_{M}(k)\right]^{T} \in \mathbb{C}^{M \times T_{0}}$. The received signal model for the $n$th AVS is as follows

$$
\mathbf{Y}_{k}^{n}=\mathbf{g}_{k}^{n}\left(\mathbf{X}_{k}\right) \mathbf{S}_{k}+\epsilon_{k}^{n}
$$

where $\mathbf{X}_{k}=\left[\mathbf{x}_{1, k}^{T}, \ldots, \mathbf{x}_{M, k}^{T}\right]^{T}$ is the source state, $\mathbf{g}_{k}^{n}\left(\mathbf{X}_{k}\right)=$ $\left[\mathbf{a}_{n}^{1}(k), \ldots, \mathbf{a}_{n}^{M}(k)\right]$ with $\mathbf{a}_{n}^{m}(k)=\left[1, \boldsymbol{u}_{m, k}^{n}\right]^{T}$ is the steering vector, and $\epsilon_{k}^{n} \in \mathbb{C}^{4 \times T_{0}}$ represent the channel noise including the pressure and velocity noise terms. Note that the particle velocity terms are normalized by multiplying by a constant term $-\rho_{0} c_{0}$, where $\rho_{0}$ and $c_{0}$ represent the ambient density and the propagation speed of the acoustic wave in the medium respectively. The noise process $\epsilon_{k}^{n}$ is a sequence of complexvalued i.i.d. circular Gaussian random variables with zero mean and covariance matrix $\Gamma$.

Since dynamic sources are considered, the source state $\mathbf{x}_{m, k}$ is constructed by cascading the original position component $\mathbf{x}_{m, k}^{\mathrm{p}}$ with a velocity component $\mathbf{x}_{m, k}^{\mathrm{v}}$. Constant velocity $(\mathrm{CV})$ model [9] is employed here to model the source dynamics as

$$
\mathbf{X}_{k}=\mathbf{F} \mathbf{X}_{k-1}+\mathbf{G} \mathbf{v}_{k}
$$

where $\mathbf{v}_{k}$ is the global uncertainties in the state process. The coefficient matrix $\mathbf{F}$ and $\mathbf{G}$ are defined respectively as

$$
\mathbf{F}=\mathbf{I}_{M} \otimes\left[\begin{array}{cc}
\mathbf{I}_{3} & \Delta T \mathbf{I}_{3} \\
\mathbf{0} & \mathbf{I}_{3}
\end{array}\right] ; \quad \mathbf{G}=\mathbf{I}_{M} \otimes\left[\begin{array}{c}
\frac{\Delta T^{2}}{2} \mathbf{I}_{3} \\
\Delta T \mathbf{I}_{3}
\end{array}\right],
$$

where $\mathbf{I}_{q}$ denotes the $q$ th order identity matrix, $\Delta T$ represents the time period in seconds between the previous and current time step, and $\otimes$ denotes the Kronecker product. Eqs. (2) and (3) present the state-space model for the AVS network based tracking problem. The nodes of the network are modeled as vertices of the graph $\mathcal{G}=(\boldsymbol{\nu}, \mathcal{E})$, namely as elements of the node set $\boldsymbol{\nu}=\{1, \ldots, N\}$. The edge set $\mathcal{E} \subseteq \boldsymbol{\nu} \times \boldsymbol{\nu}$ represents the network's communication constraints, i.e., if node $n$ can send information to node $u$ then $(n, u) \in \mathcal{E}$. For graph $\mathcal{G}$, the maximum degree $\Delta_{\mathcal{G}}=\max _{n} D^{(n)}$, where $D^{(n)}$ is the number of neighboring nodes for node $n$. We present the proposed distributed acoustic source tracking algorithm for the AVS network in the next two sections.

\section{Estimation OF LocAl STATISTICS}

The AVS network uses a large number of snapshots at each time step which leads to the covariance matrix of large dimensions. Therefore, the unscented particle filter (UPF) [10], [11] and the cEIPF [9], typically used for centralized nonlinear tracking, are computationally demanding and not suitable for distributed acoustic source tracking using the AVS network. In this letter, a distributed unscented information particle filter (dUIPF) is developed, which reduces the size of the covariance matrix computed, and keeps the tracking algorithm computationally practical. In comparison with the cEIPF [9], the dUIPF does not require a $\mathrm{CP}$ and eliminates the need for computation of a large Hessian matrix.

The proposed dUIPF updates the Fisher information matrix $\widetilde{\mathcal{I}}_{k-1}^{n} \triangleq\left(\widetilde{\mathbf{P}}_{k-1}^{n}\right)^{-1}$ and the information state vector $\widetilde{\mathcal{Z}}_{k-1}^{n} \triangleq$ $\widetilde{\mathcal{I}}_{k-1}^{n} \widetilde{\mathbf{X}}_{k-1}^{n}$ at each local AVS node instead of the source state $\widetilde{\mathbf{X}}_{k-1}^{n}$ and the covariance matrix $\widetilde{\mathbf{P}}_{k-1}^{n}$. Starting at the conclusion of iteration $(k-1)$, the global source state estimate $\widehat{\mathbf{X}}_{k-1}^{n}$ and the global covariance estimate $\widehat{\mathbf{P}}_{k-1}^{n}$ (Step 8 below) are available. The $k$ th iteration of the dUIPF is explained next.

Step 1: A set of sigma points is calculated at node $n$ as follows

$$
\boldsymbol{\mathcal { X }}_{k-1}^{n, j}=\widehat{\mathbf{X}}_{k-1}^{n} \pm\left\{\sqrt{\left(n_{x}+\kappa\right) \widehat{\mathbf{P}}_{k-1}^{n}}\right\}^{j},
$$

where $\kappa$ is a scaling parameter, $n_{x}$ is the dimension of $\widehat{\mathbf{X}}_{k-1}^{n}$, and term $\left\{\sqrt{\left(n_{x}+\kappa\right) \widehat{\mathbf{P}}_{k-1}^{n}}\right\}^{j}$ corresponds to the $j$ th column of the square root of matrix $\left(n_{x}+\kappa\right) \widehat{\mathbf{P}}_{k-1}^{n}$. The initial condition is given by $\mathcal{X}_{k}^{n, 0}=\widehat{\mathbf{X}}_{k-1}^{n}$. The associated weights for the sigma points are $\mathcal{W}^{j}=1 /\left(2\left(n_{x}+\kappa\right)\right)$ with the following initial condition $\mathcal{W}^{0}=\kappa /\left(n_{x}+\kappa\right)$.

Step 2: Sigma points are updated based on (3) as follows

$$
\mathcal{X}_{k \mid k-1}^{n, j}=\mathbf{F} \mathcal{X}_{k-1}^{n, j}+\mathbf{G v}_{k}, \quad \text { for } j=0, \ldots, 2 n_{x} .
$$

The predicted measurements based on these sigma points are

$$
\mathcal{Y}_{k \mid k-1}^{n, j}=\mathbf{g}_{k}^{n}\left(\mathcal{X}_{k \mid k-1}^{n, j}\right) \overline{\mathbf{S}}_{k}^{n}, \quad \text { for } j=0, \ldots, 2 n_{x},
$$

where $\overline{\mathbf{S}}_{k}^{n}$ is maximum likelihood estimate of the source signal,

$\overline{\mathbf{S}}_{k}^{n}=\left(\left(\mathbf{g}_{k}^{n}\left(\boldsymbol{\mathcal { X }}_{k \mid k-1}^{n, j}\right)\right)^{H} \mathbf{g}_{k}^{n}\left(\mathcal{X}_{k \mid k-1}^{n, j}\right)\right)^{-1}\left(\mathbf{g}_{k}^{n}\left(\boldsymbol{\mathcal { X }}_{k \mid k-1}^{n, j}\right)\right) \mathbf{Y}_{k}^{n}$,

with superscript $H$ denoting the Hermitian operator. The predicted source state, the error covariance matrix, and the cross-covariance are, respectively, obtained as

$$
\begin{aligned}
\overline{\mathbf{X}}_{k \mid k-1}^{n} & =\sum_{j=0}^{2 n_{x}} \mathcal{W}^{j} \mathcal{X}_{k \mid k-1}^{n, j}, \\
\overline{\mathbf{P}}_{k \mid k-1}^{n} & =\sum_{j=0}^{2 n_{x}} \mathcal{W}^{j} \widetilde{X}_{k}^{n, j}\left(\widetilde{X}_{k}^{n, j}\right)^{T}, \\
\text { and } \quad \overline{\mathbf{P}}_{k \mid k-1}^{n, \mathcal{Y} \mathcal{Y}} & =\sum_{j=0}^{2 n_{x}} \mathcal{W}^{j} \tilde{X}_{k}^{n, j}\left(\tilde{Y}_{k}^{n, j}\right)^{T},
\end{aligned}
$$

where $\tilde{X}_{k}^{n, j}=\mathcal{X}_{k \mid k-1}^{n, j}-\overline{\mathbf{X}}_{k \mid k-1}^{n}$ and $\tilde{Y}_{k}^{n, j}=\mathcal{Y}_{k \mid k-1}^{n, j}-\mathbf{Y}_{k}^{n}$. 
Step 3: The predicted information matrix and state vector are

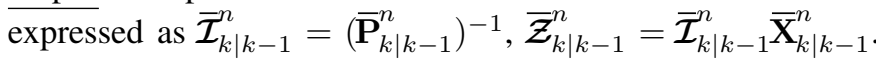
The information contribution equations are given by

$$
\begin{aligned}
\mathcal{K}_{k}^{n} & =\left(\mathbf{H}_{k}^{n}\right)^{T}\left(\mathbf{R}_{k}^{n}\right)^{-1}\left(\boldsymbol{\Xi}_{k}^{n}+\mathbf{H}_{k}^{n} \overline{\mathbf{X}}_{k \mid k-1}^{n}\right), \\
\mathcal{G}_{k}^{n} & =\left(\mathbf{H}_{k}^{n}\right)^{T}\left(\mathbf{R}_{k}^{n}\right)^{-1} \mathbf{H}_{k}^{n}
\end{aligned}
$$

where $\left(\mathbf{H}_{k}^{n}\right)^{T}=\left(\overline{\mathbf{P}}_{k \mid k-1}^{n}\right)^{-1} \overline{\mathbf{P}}_{k \mid k-1}^{n, \mathcal{X} \mathcal{Y}}$,

$$
\boldsymbol{\Xi}_{k}^{n}=\mathbf{Y}_{k}^{n}-\mathbf{g}_{k}^{n}\left(\overline{\mathbf{X}}_{k \mid k-1}^{n}\right) \overline{\mathbf{S}}_{k}^{n}
$$

Step 4: The Fisher information matrix and the information

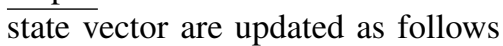

$$
\widetilde{\mathcal{I}}_{k}^{n}=\mathcal{K}_{k}^{n}+\overline{\mathcal{I}}_{k \mid k-1}^{n}, \quad \widetilde{\mathcal{Z}}_{k}^{n}=\mathcal{G}_{k}^{n}+\overline{\mathcal{Z}}_{k \mid k-1}^{n} .
$$

The dUIPF incorporates the global state statistics from the previous iteration $\left(\widehat{\mathbf{X}}_{k-1}^{n}\right.$ and $\left.\widehat{\mathbf{P}}_{k-1}^{n}\right)$ into Step 1 to compute $\widetilde{\mathcal{I}}_{k-1}^{n}$ and $\widetilde{\mathcal{Z}}_{k-1}^{n}$ (Step 4), which are then used below in Step 5.

Step 5: The dUIPF computation savings come at this step. $\overline{\text { Local }} \mathrm{PF}$ at node $n$, uses $\widetilde{\mathcal{I}}_{k-1}^{n}$ and $\widetilde{\mathcal{Z}}_{k-1}^{n}$ (computed at Step 4) to approximate the optimal importance function $q\left(\mathbf{X}_{k}^{n} \mid \mathbf{X}_{k-1}^{n}, \mathbf{Y}_{k}^{n}\right)$, i.e., the particles are generated as follows

$$
\mathbf{X}_{k}^{n,(\ell)} \sim q\left(\mathbf{X}_{k}^{n} \mid \mathbf{X}_{k-1}^{n}, \mathbf{Y}_{k}^{n}\right)=N\left(\widetilde{\mathbf{X}}_{k}^{n}, \widetilde{\mathbf{P}}_{k}^{n}\right),
$$

where $\quad \widetilde{\mathbf{P}}_{k}^{n}=\left(\widetilde{\mathcal{I}}_{k}^{n}\right)^{-1}, \quad$ and $\quad \widetilde{\mathbf{X}}_{k}^{n}=\widetilde{\mathbf{P}}_{k}^{n} \widetilde{\mathcal{Z}}_{k}^{n}$.

Note that the size of the matrix to be inverted in the dUIPF is of $\mathrm{O}(6 M \times 6 M)$, while for the UPF, the size of the matrix to be inverted is of $\mathrm{O}\left(4 N T_{0} \times 4 N T_{0}\right)$ where $4 N T_{0} \gg 6 M$.

Step 6: The importance weights at node $n$ are calculated as

$$
w_{k}^{n,(\ell)}=w_{k-1}^{n,(\ell)} \frac{p\left(\mathbf{Y}_{k}^{n} \mid \mathbf{X}_{k}^{n,(\ell)}\right) p\left(\mathbf{X}_{k}^{n,(\ell)} \mid \mathbf{X}_{k-1}^{n,(\ell)}\right)}{q\left(\mathbf{X}_{k}^{n,(\ell)} \mid \mathbf{X}_{k-1}^{n,(\ell)}, \mathbf{Y}_{k}^{n}\right)}
$$

where transition density $p\left(\mathbf{X}_{k}^{n,(\ell)} \mid \mathbf{X}_{k-1}^{n,(\ell)}\right)$ and likelihood $p\left(\mathbf{Y}_{k}^{n} \mid \mathbf{X}_{k}^{n,(\ell)}\right)$ are obtained from (3) and (2) respectively.

Step 7: After resampling, the minimum mean square error

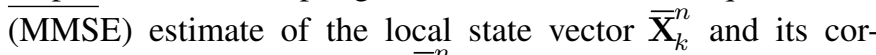
responding error covariance $\overline{\mathbf{P}}_{k}^{n}$ is computed as

$$
\begin{aligned}
& \overline{\mathbf{X}}_{k}^{n}=\sum_{\ell=1}^{L} w_{k}^{n,(\ell)} \mathbf{X}_{k}^{n,(\ell)} \\
& \overline{\mathbf{P}}_{k}^{n}=\sum_{\ell=1}^{L} w_{k}^{n,(\ell)}\left(\mathbf{X}_{k}^{n,(\ell)}-\overline{\mathbf{X}}_{k}^{n}\right)\left(\mathbf{X}_{k}^{n,(\ell)}-\overline{\mathbf{X}}_{k}^{n}\right)^{T}
\end{aligned}
$$

To summarize, Steps 1 to 4 of the dUIPF compute the local Fisher information matrix $\widetilde{\mathcal{I}}_{k}^{n}$ and information vector $\widetilde{\mathcal{Z}}_{k}^{n}$ at node $n$ using the previous global statistics $\left(\widehat{\mathbf{X}}_{k-1}^{n}\right.$ and $\left.\widehat{\mathbf{P}}_{k-1}^{n}\right)$ to be used as the proposal distribution at local PFs (Steps 5 to 7). The next step of the dUIPF, Step 8 introduced below, is to fuse local statistics to obtain the global state estimates.

\section{DISTRIBUTED INFORMATION FUSION}

Step 8: The local particles and their associated weights are

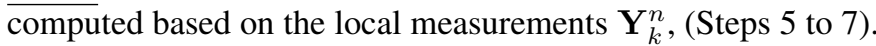
The local state estimates $\mathbb{E}\left(\mathbf{X}_{k}^{n} \mid \mathbf{Y}_{k}^{n}\right)$ are, therefore, localized and different across the AVS network. In order to provide consistency among the local estimates, an information fusion step is introduced to combine local statistics $\overline{\mathbf{X}}_{k}^{n}, \overline{\mathbf{P}}_{k}^{n}, \overline{\mathcal{Z}}_{k \mid k-1}^{n}$, and $\overline{\mathcal{I}}_{k \mid k-1}^{n}$ into a common set of global statistics $\widehat{\mathbf{X}}_{k}^{n}$ and $\widehat{\mathbf{P}}_{k}^{n}$ across the network. Based on the Chong-Mori-Chang trackfusion theorem [12], the following fusion rules are derived

$$
\begin{aligned}
{\left[\widehat{\mathbf{P}}_{k}^{n}\right]^{-1} } & =\overline{\mathcal{I}}_{k \mid k-1}^{n}+\underbrace{\sum_{n=1}^{N}\left\{\left[\overline{\mathbf{P}}_{k}^{n}\right]^{-1}-\overline{\mathcal{I}}_{k \mid k-1}^{n}\right\}}_{\boldsymbol{P}_{c}(\infty)} \\
\widehat{\mathbf{X}}_{k}^{n} & =\left[\widehat{\mathbf{P}}_{k}^{n}\right]^{-1}[\overline{\mathcal{Z}}_{k \mid k-1}^{n}+\underbrace{\sum_{n=1}^{N}\left\{\left[\overline{\mathbf{P}}_{k}^{n}\right]^{-1} \overline{\mathbf{X}}_{k}^{n}-\overline{\mathcal{Z}}_{k \mid k-1}^{n}\right\}}_{\mathbf{x}_{c}(\infty)}]
\end{aligned}
$$

Using average consensus algorithms [11], $\left\{\mathbf{x}_{c}(\infty), \boldsymbol{P}_{c}(\infty)\right\}$ are obtained by iterating the following consensus rules

$$
\begin{aligned}
& \mathbf{X}_{c}^{n}(t+1)=\mathbf{X}_{c}^{n}(t)+\epsilon \sum_{j \in \aleph^{(n)}}\left(\mathbf{X}_{c}^{j}(t)-\mathbf{X}_{c}^{n}(t)\right), \\
& \mathbf{P}_{c}^{n}(t+1)=\mathbf{P}_{c}^{n}(t)+\epsilon \sum_{j \in \aleph^{(n)}}\left(\mathbf{P}_{c}^{j}(t)-\mathbf{P}_{c}^{n}(t)\right),
\end{aligned}
$$

where $\epsilon \in\left(0,1 / \Delta_{\mathcal{G}}\right)$ with the following initial conditions

$$
\begin{aligned}
& \mathbf{P}_{c}^{n}(t=0)=\left[\overline{\mathbf{P}}_{k}^{n}\right]^{-1}-\overline{\mathcal{I}}_{k \mid k-1}^{n}, \\
& \mathbf{X}_{c}^{n}(t=0)=\left[\overline{\mathbf{P}}_{k}^{n}\right]^{-1} \overline{\mathbf{X}}_{k}^{n}-\overline{\mathcal{Z}}_{k \mid k-1}^{n} .
\end{aligned}
$$

Eqs. (23) and (24) converge to $\left\{\mathbf{X}_{c}(\infty), \mathbf{P}_{c}(\infty)\right\}$ after convergence of the consensus algorithm. The consensus rules in Eqs. (23) and (24) are distributed where communication is limited to local neighborhood. After a burn-in period, the global estimation of source state $\widehat{\mathbf{X}}_{k}^{n}$ and corresponding covariance $\widehat{\mathbf{P}}_{k}^{n}$ can be obtained.

\section{Simulations}

Both single source tracking and multiple source tracking scenarios are organized to study the performance of the proposed dUIPF approach. Two different source trajectories are simulated: one is from $(-10,-40,-20) \mathrm{m}$ to $(-100,60,-120) \mathrm{m}$, and the other from $(110,80,20) \mathrm{m}$ to $(10,-20,120) \mathrm{m}$ with 30 time steps. For single source tracking, only the first source is considered. For multiple source tracking, both sources are simultaneously active. Such motions result in a velocity of $\pm 3.5 \mathrm{~m} / \mathrm{s}$ roughly along each coordinate axis. 15 AVSs are deployed to formulate a distributed AVS network. The sensor locations are randomly drawn in the 3-D space. The wideband source signals are uncorrelated from each other and from one snapshot to the next so that each signal has a flat spectral density and its bandwidth equals the sampling frequency. The background noise (evaluated by the signal to 

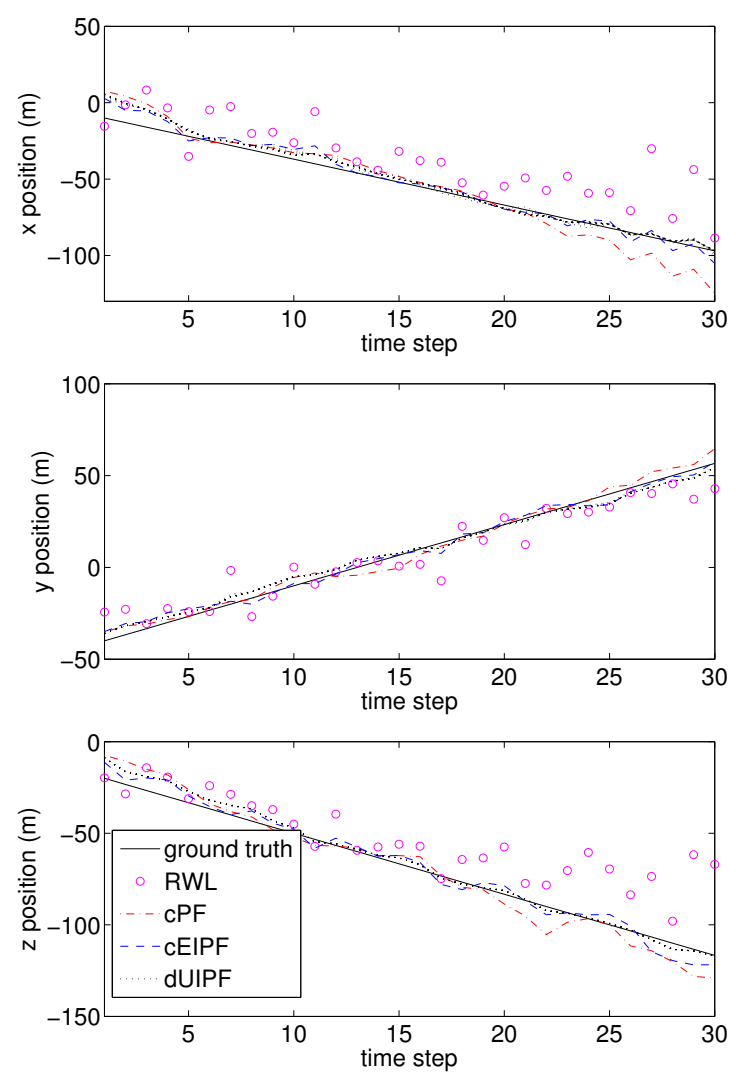

Fig. 1. Tracking results of $x-$ (up), $y-$ (middle), and $z-$ (bottom) coordinate under $\mathrm{SNR}=0 \mathrm{~dB}$ and $T_{0}=8$ for (a) single source and (b) two sources.

noise ratio (SNR)) is simulated by adding the complex circular white Gaussian noise to the received signal. The tracking performance of the proposed dUIPF approach is compared with that using RWL localization algorithm [1], cPF tracking algorithm [8] and cEIPF tracking algorithm [9]. The cPF tracking algorithm is not available in a straightforward manner for the AVS based tracking problem here. We have replaced the pseudo likelihood in [8] by the Capon beamforming response [8] for single source case and concentrated likelihood [9] for multiple source case. It is worth mentioning that the RWL approach cannot be employed for multiple source localization scenario since it is difficult to associate the DOA with the correct source. In our implementation, we assume perfect DOA association is available for the RWL algorithm, i.e., the measured DOAs are associated with ground truth DOA of each source. The RWL approach is then applied to estimate the source positions. The parameters for proposed dUIPF are set to: $L=500, \boldsymbol{\Sigma}_{v}=0.01 \mathbf{I}_{3 M}, \kappa=0.5$ and $\boldsymbol{\Gamma}=50 \mathbf{I}_{4 T_{0}}$. This parameter setup is found adequate for the following simulations. The source velocities are initialized around the ground truth. The initial positions are coarsely estimated by using a maximum likelihood estimator.

Figure 1 and Fig. 2 show the 3-D position estimation results from a single implementation under $\mathrm{SNR}=0 \mathrm{~dB}$ and $T_{0}=8$. Under both single source and two sources scenario, the dUIPF
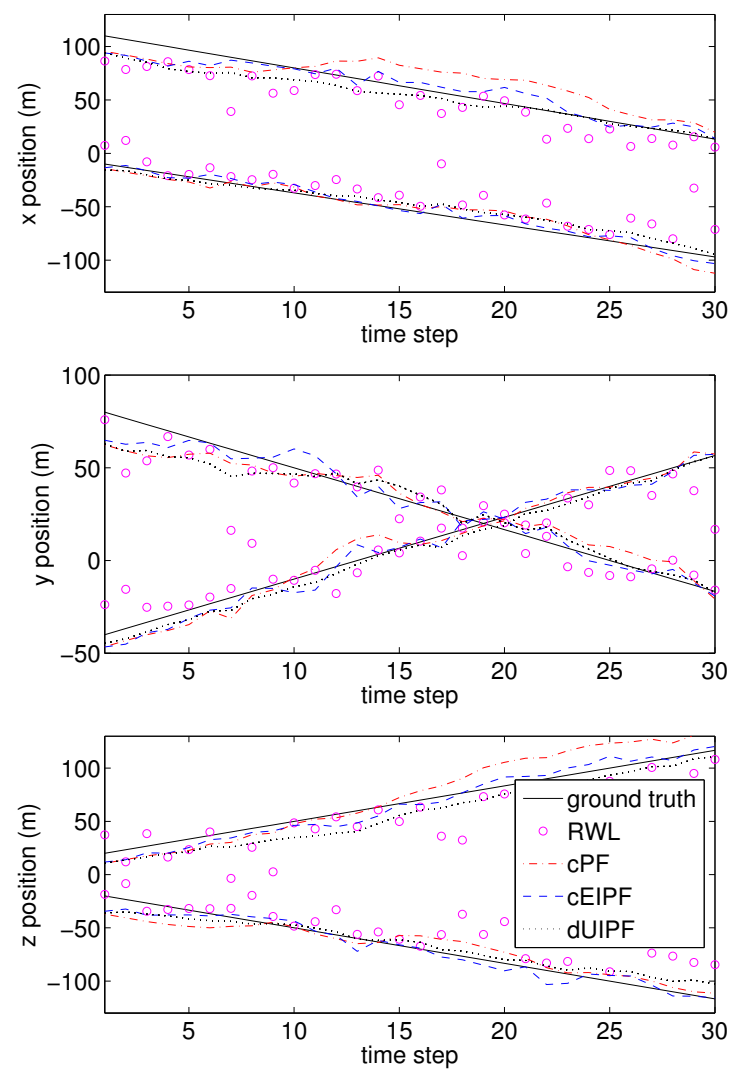

Fig. 2. Tracking results of $x-$ (up), $y-$ (middle), and $z-$ (bottom) coordinate under $\mathrm{SNR}=0 \mathrm{~dB}$ and $T_{0}=8$ for (a) single source and (b) two sources.

is able to track the trajectories of sources accurately. The tracking result of the dUIPF is significantly better than that of the RWL and cPF approaches. It is also noted that the tracking result of the dUIPF is similar to that of its centralized counterpart (cEIPF). To evaluate the average performance, the root mean square error (RMSE) over 50 Monte Carlo (MC) runs under different SNRs and different numbers of snapshots for single source and multiple sources are presented in Fig. 3 and Fig. 4, respectively. The number of snapshots is fixed to 8 for studying the performance under different SNRs and the SNR is fixed to $0 \mathrm{~dB}$ for studying the performance under different numbers of snapshots. The results show that the proposed dUIPF is able to provide good tracking accuracy for multiple sources as well as single source. It performs much better than the RWL and cPF approaches, and its performance is favorably close to the cEIPF. When the SNR is relatively high and the number of snapshots is relatively large, the performance of the proposed dUIPF is virtually overlaps with that of the cEIPF. However, due to distributed implementation, both communication and computation cost of the dUIPF are lower than the cEIPF.

\section{CONCLUSIONS}

A distributed tracking approach for 3-D source tracking using an AVS network is proposed in this paper. The pro- 

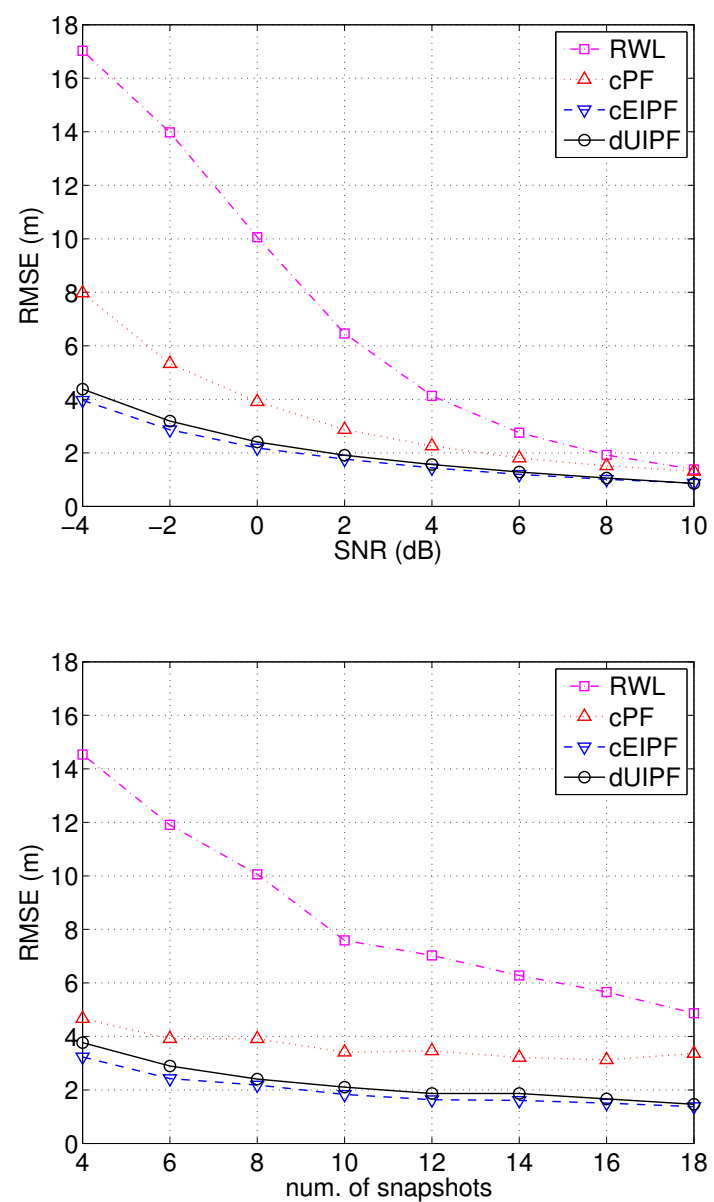

(b)

Fig. 3. RMSE over $50 \mathrm{MC}$ runs for single source tracking versus different SNRs.

posed dUIPF is able to directly fuse the information from the received signals and track multiple wideband acoustic sources. It outperforms the RWL localization approach [1] and centralized PF approach [8]. Also, the performance of the dUIPF is favorably comparable with that of cEIPF [9] when SNR is relatively high and the number of snapshots is relatively large (e.g., SNR $>2 \mathrm{~dB}$ and $T_{0}>10$ ). The performance bound and applications of the proposed approach in real acoustic environments will be studied in future works.

\section{REFERENCES}

[1] M. Hawkes and A. Nehorai, "Wideband source localization using a distributed acoustic vector-sensor array," IEEE Trans. Signal Process., vol. 51, no. 6, pp. 1479-1491, 2003.

[2] V. N. Hari, G. V. Anand, A. B. Premkumar, and A. S. Madhukumar, "Design and performance analysis of a signal detector based on suprathreshold stochastic resonance," Signal Process., vol. 92, no. 7, pp. 1745-1757, 2012.

[3] X. Zhong, A. B. Premkumar, and A. S. Madhukumar, "Particle filtering and posterior Cramér-Rao bound for 2-D direction of arrival tracking using an acoustic vector sensor," IEEE Sensors J., vol. 12, no. 2, pp. 363-377, 2012.

[4] X. Zhong and A. B. Premkumar, "Particle filtering approaches for multiple acoustic source detection and 2-D direction of arrival estimation
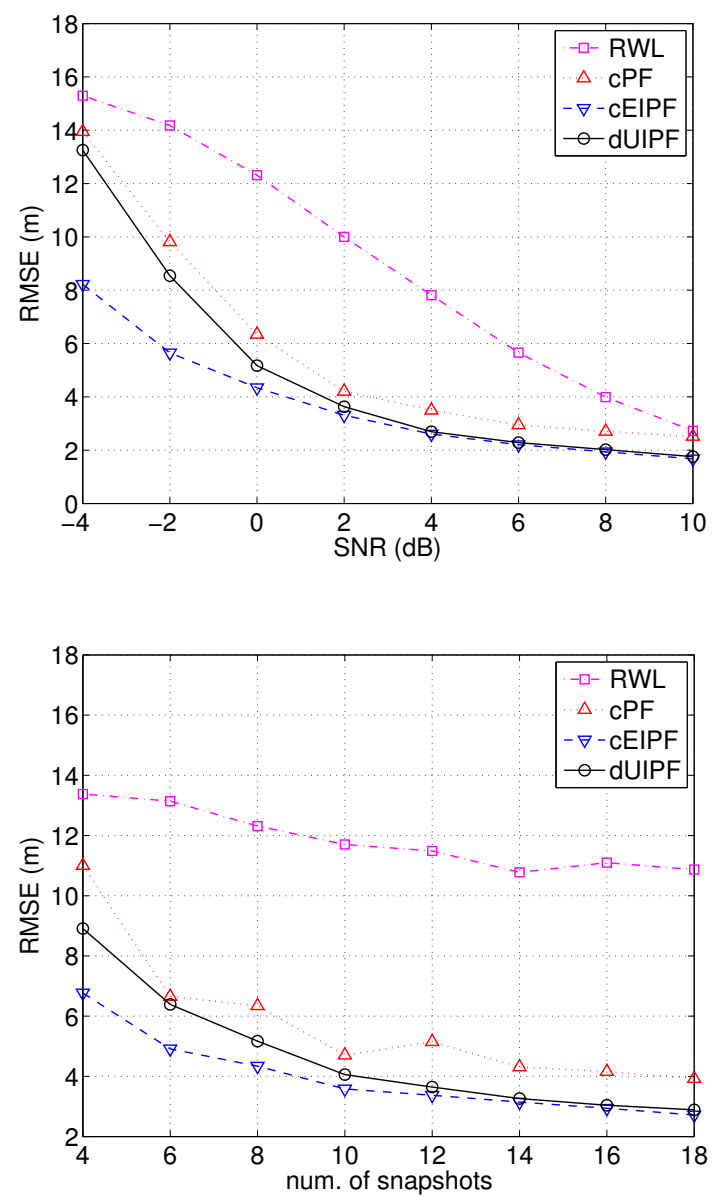

(b)

Fig. 4. RMSE over $50 \mathrm{MC}$ runs for multiple source tracking versus different numbers of snapshots.

using a single acoustic vector sensor," IEEE Trans. Signal Process., vol. 60, no. 9, pp. $4719-4733,2012$.

[5] D. Levin, E. A. P. Habets, and S. Gannot, "On the angular error of intensity vector based direction of arrival estimation in reverberant sound fields," J. Acoust. Soc. Amer., vol. 128, no. 4, pp. 1800-1811, 2010.

[6] A. Song, A. Abdi, M. Badiey, and P. Hursky, "Experimental demonstration of underwater acoustic communication by vector sensors," IEEE J. Ocean. Eng., vol. 36, no. 3, pp. 454-461, 2011.

[7] Deok-Jin Lee, "Nonlinear estimation and multiple sensor fusion using unscented information filtering," IEEE Signal Process. Lett., vol. 15, pp. $861-864,2008$

[8] D.B. Ward, E.A. Lehmann, and R.C. Williamson, "Particle filtering algorithms for tracking an acoustic source in a reverberant environment," IEEE Trans. Speech Audio Process., vol. 11, no. 6, pp. 826-836, 2003.

[9] X. Zhong, A. B. Premkumar, and C.-T. Lau, "An extended information pf for wideband acoustic source tracking using a distributed AVS array," in Proc. 20th Conf. European Signal Process., pp. 1319-1323, 2012.

[10] A. Mohammadi and A. Asif, "A constraint sufficient statistics based distributed particle filter for bearing only tracking," in Proc. IEEE Int. Conf. Commun., pp. 3670-3675, 2012.

[11] A. Mohammadi and A. Asif, "Distributed particle filter implementation with intermittent/irregular consensus convergence," IEEE Trans. Signal Process., In press 2013.

[12] C.Y. Chong, S. Mori, and K.C. Chang, Multitarget-Multisensor Tracking: Advanced Applications, chapter Distributed Multitarget Multisensor Tracking, Artech House, 1990. 